\title{
Half Fourier Acquisition Applied to Time Series Analysis of Contrast Agent Uptake
}

\author{
Andreas Degenhard ${ }^{1}$, Christine Tanner ${ }^{2}$, Carmel Hayes ${ }^{1}$, David J. Hawkes ${ }^{2}$, \\ and Martin O. Leach ${ }^{1}$ \\ 1 CRC Clinical MR Research Group \\ The Institute of Cancer Research and the Royal Marsden NHS Trust \\ Sutton, Surrey SM2 5PT, UK \\ andreasd@icr.ac.uk \\ 2 Division of Radiological Sciences and Medical Engineering \\ The Guy's, King's and St. Thomas' School of Medicine and Dentistry \\ Thomas Guy House, Guy's Hospital, London SE1 9RT, UK
}

\begin{abstract}
Magnetic Resonance Imaging (MRI) has emerged as a powerful tool in medical diagnosis and research. Dynamic Contrast Enhanced MRI (DCE-MRI), which involves the administration of a paramagnetic contrast medium, has been shown to provide additional sensitivity in detecting abnormality. In particular, in imaging of the breast and axilla the ability to differentiate between benign and malignant lesions depends, in part, on the enhancement profile. Evaluation of this requires a sufficient temporal resolution in the time series acquisition. Faster imaging in general requires that images are acquired with a lower spatial resolution or signal to noise ratio. In this paper we investigate a novel approach for increasing the temporal resolution in DCE-MRI without decreasing the accuracy of the measurement. The results clearly indicate a superior image quality compared to standard half Fourier techniques and the algorithm correctly recovers the time series characteristics of $T_{1}$ and $T_{2}^{*}$-weighted time series data.
\end{abstract}

\section{Introduction}

Magnetic Resonance Imaging (MRI) is a valuable imaging modality because of its high contrast sensitivity to many characteristics of tissues and body fluids, including proton density, $T_{1}$ and $T_{2}$ relaxation times. Dynamic Contrast Enhanced MRI (DCE-MRI) using a paramagnetic contrast medium with a non-selective extracellular distribution, such as gadolinium diethylene triamine pentaacetic acid (Gd-DTPA), is currently essential in the detection and evaluation of breast cancer with MRI [12]. Observation of contrast enhancement is typically achieved using dynamic imaging techniques where the contrast agent is injected during acquisition of a dynamic series of images. Following contrast agent administration, malignant breast lesions tend to enhance more rapidly than benign lesions, a phenomenon also seen in imaging involving lymph nodes [4]. 
In this work we apply dynamic acquisition techniques to $T_{1}$ and $T_{2}^{*}$-weighted MR imaging. Both imaging methods have been investigated for differentiating between benign and malignant breast [3] and brain lesions 7]. For an insufficient temporal resolution time-feature characteristics such as peak-enhancement or first pass perfusion drop are not accurately characterized. Furthermore, successful pharmacokinetic modeling of time series data depends on the numerical stability in the fitting procedure and this in turn depends on the number of available data points describing the enhancement profile [6].

Partial Fourier acquisition techniques improve the temporal resolution, although the reconstructed images suffer from a reduced signal to noise ratio, inferior spatial resolution or reconstruction artifacts affecting the accuracy of the time series data. In this work we present an image reconstruction algorithm based on the concepts of image recovery theory 9] which approximately doubles the temporal resolution. The proposed algorithm requires a full Fourier Space (FS) acquisition of a pre-contrast image, that is, prior to contrast agent administration. To test our algorithm, half Fourier data have been generated from a full post-contrast FS acquisition for two reasons: Firstly the fully acquired FS data provides the original or true post-contrast image which is then used as a reference image to evaluate the image quality of the reconstructed images in the time series. Secondly the full post-contrast phase data are used in the comparison of reconstruction methods. Phase retrieval algorithms applied to half Fourier data can introduce phase errors which interfere with the performance of the different constrained amplitude reconstruction algorithms [9].

\section{Methods and Material}

MR data are acquired in Fourier space (FS) and the corresponding real space (RS) image is calculated by a linear map, i.e. the Inverse Fourier Transform (IFT) [8]. According to the Hermitian symmetry of FS, the bulk of the signal intensities (SI) are distributed symmetrically within the centre or low frequency regime of FS, whereas most of the information about the shape and the structure of the RS image is included in the outer high frequency part 8 .

In a MR image acquisition, the pixel intensity in the RS image is also a complex value, since motion and magnetic field inhomogeneities introduce a nonzero or nontrivial phase to the RS image data. The nontrivial phase structure in the RS image leads to a deviation from the perfect symmetry in FS. Nevertheless images may be reconstructed from a half sampled FS data set using Fourier reconstruction methods [5]. Half Fourier acquisition techniques sample approximately half of the complete FS data as shown in Figure 1(a), thus decreasing the image acquisition time. However, an IFT applied to the undersampled FS results in poor quality RS images and constrained methods are the mathematical tools developed to provide additional information required to restore unsampled FS data. In this paper we compare two standard phase constrained reconstruction methods, the Margosian method [5] and a so-called projection on convex sets technique (POCS) [9], with two new variations on the latter summarized as 
(a) Margosian \& POCS method

\begin{tabular}{|c:c|}
\hline Sampled & Zero \\
fraction of & filling \\
Fourier space & \\
& \\
\hline
\end{tabular}

(b) Pre-processed POCS method

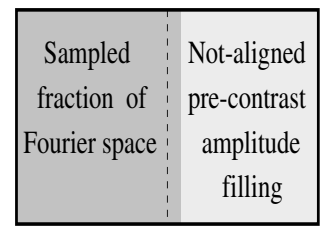

(c) Pre-processed POCS method

\begin{tabular}{|c:c|}
\hline Sampled & Aligned \\
fraction of & pre-contrast \\
Fourier space & amplitude \\
& filling \\
\hline
\end{tabular}

Fig. 1. In the Margosian and the POCS method the unsampled fraction of FS is filled with zeros. For the pre-processed POCS method two possible filling procedures are shown. One half of FS and eight additional lines from the centre of FS are sampled.

pre-processed POCS methods. Both the Margosian and the POCS method are based on initial zero-filling of the unsampled half of FS and use the post-contrast phase to force or align the amplitude RS image according to the desired phase structure [5]. Unlike the Margosian approach, in the POCS method the postcontrast phase information is used iteratively to accomplish a sequence of phase constrained symmetrization steps. Each of the symmetrization steps starts with inserting the desired post-contrast phase information followed by a Fourier transform in RS or an inverse Fourier transform in FS to align the amplitude data to the post-contrast phase. Both methods require the acquisition of additional low frequency FS data for Hamming filtering to provide a smooth transition to the zero-filled part [5]. In the pre-processed POCS method, the unsampled half of FS is filled with pre-contrast amplitude data acquired prior to the dynamic series as schematically shown in Figure 1(b) and no Hamming filtering needs to be applied. The pre-contrast data can be aligned to the post-contrast phase using one or more symmetrization steps as in the conventional POCS method (c). After the unsampled half has been filled with unaligned or aligned pre-contrast data the conventional POCS method is applied.

In order to quantify the image quality of the different reconstruction methods the root-mean-square error (RMSE) is calculated by [6]

$$
\operatorname{RMSE}\left(S^{*}\right)=\sqrt{\frac{1}{N} \cdot\left[\sum_{i=1}^{N}\left(S_{i}^{*}-S_{i}\right)^{2}-\frac{1}{N}\left(\sum_{i=1}^{N} S_{i}^{*}-S_{i}\right)^{2}\right]} .
$$

In equation (11), $N$ denotes the total number of pixels in the image and $S_{i}$ and $S_{i}^{*}$ denote the SI of a pixel in the true image $S$ and the reconstructed image $S^{*}$ respectively.

Images were acquired on a 1.5 Tesla Siemens Vision MR System. For the $T_{1^{-}}$ weighted dynamic imaging of the breast, a 3D fast gradient echo sequence was used with $\mathrm{TR}=12 \mathrm{~ms}, \mathrm{TE}=5 \mathrm{~ms}$, flip angle $=35^{\circ}, \mathrm{FOV}=340 \mathrm{~mm}$ and spatial resolution of $1.33 \mathrm{~mm} \times 1.33 \mathrm{~mm} \times 2.5 \mathrm{~mm}(256 \times 128 \times 64$ voxels $) . T_{2}^{*}$-weighted MR images of the brain with a matrix size of $256 \times 256$ pixels were acquired with $\mathrm{TR}=26.5 \mathrm{~ms}, \mathrm{TE}=20 \mathrm{~ms}$, flip angle $=15^{\circ}$ and $\mathrm{FOV}=250 \mathrm{~mm}$. The 
acquisition time of an image is 90 secs for the $3 \mathrm{D}$ and 11 secs for the $2 \mathrm{D}$ dynamic sequence.

(a)
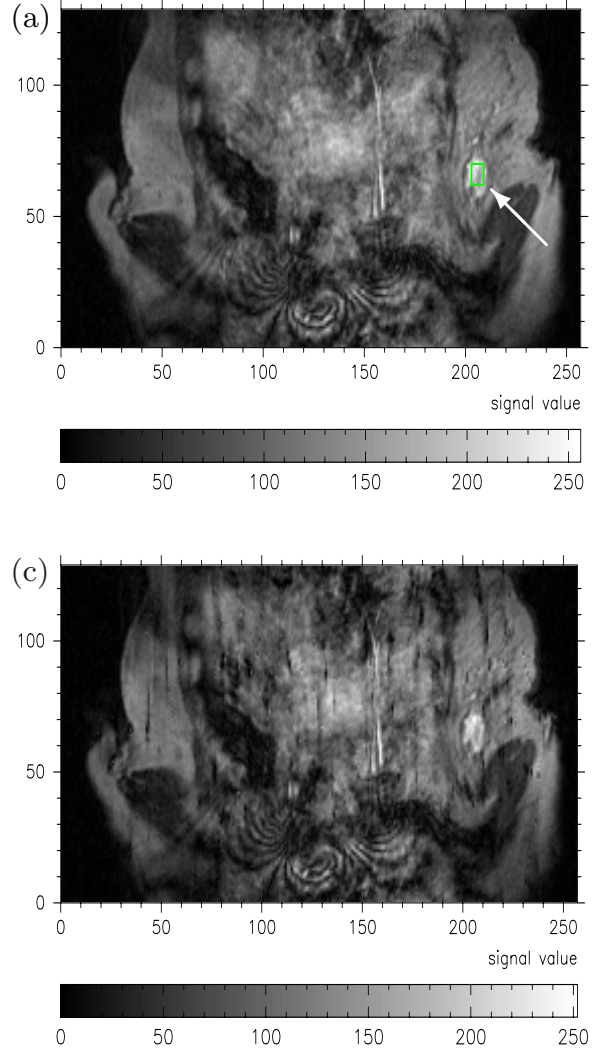
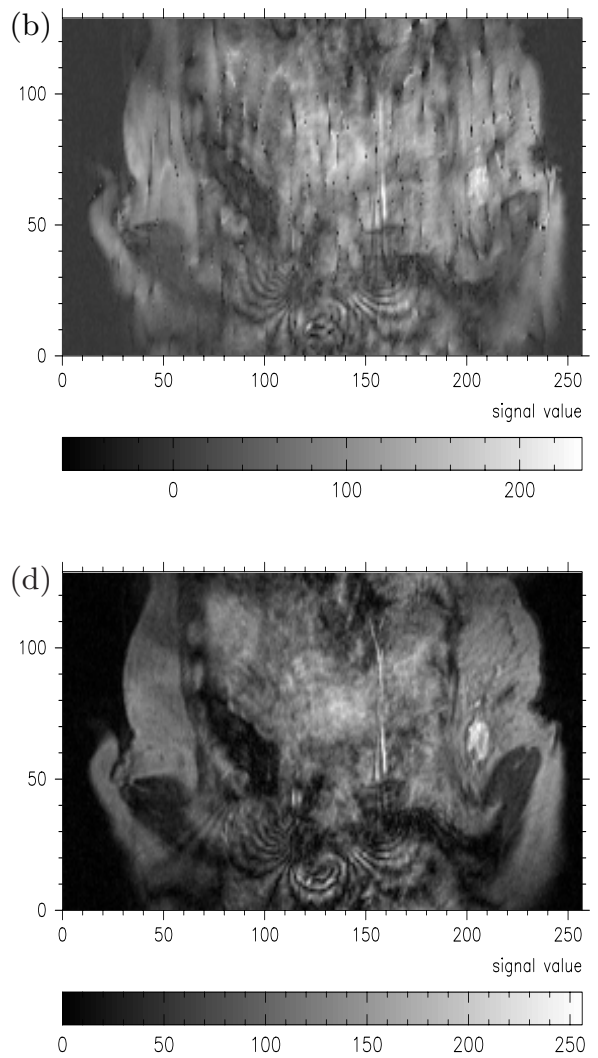

Fig. 2. A true post-contrast breast MR image showing the anatomy behind the breast wall including the axilla (a). The arrow is pointing to a malignant lymph node in the true image including a selected region of interest (ROI). Half Fourier reconstructed MR images are shown generated by the Margosian method (b) and the POCS method (c) using eight additional lines in the centre of FS. The image reconstructed using the pre-processed POCS method with aligned pre-contrast amplitude filling and eight additional lines in the centre of FS (d) shows no visible differences by comparing with the true image (a). The images for the conventional and the pre-processed POCS method are reconstructed using five final POCS iterations.

\section{Results}

Figure 2(a) shows a coronal slice of a $T_{1}$-weighted $3 \mathrm{D}$ breast MR image after contrast agent injection. A malignant lymph node (white arrow) has enhanced 
within the left breast and a ROI (63 pixels) was chosen from the true image Figure 2(a) for further time series analysis. Figure 2(b) and (c) show images reconstructed by the Margosian and the POCS method for the same slice using approximately half of the acquired full FS. For both reconstruction methods the eight additional lines in the centre of FS were used for asymmetric Hamming filtering providing a smooth transition to the zero-filled part [5]. Although it is clear from visual inspection that the POCS method has resulted in a considerable improvement in image quality, both images suffer from different types of artifacts not visible in the true image Figure 2 (a). Increasing the number of iterations in the POCS method does not correct for the large reconstruction errors.
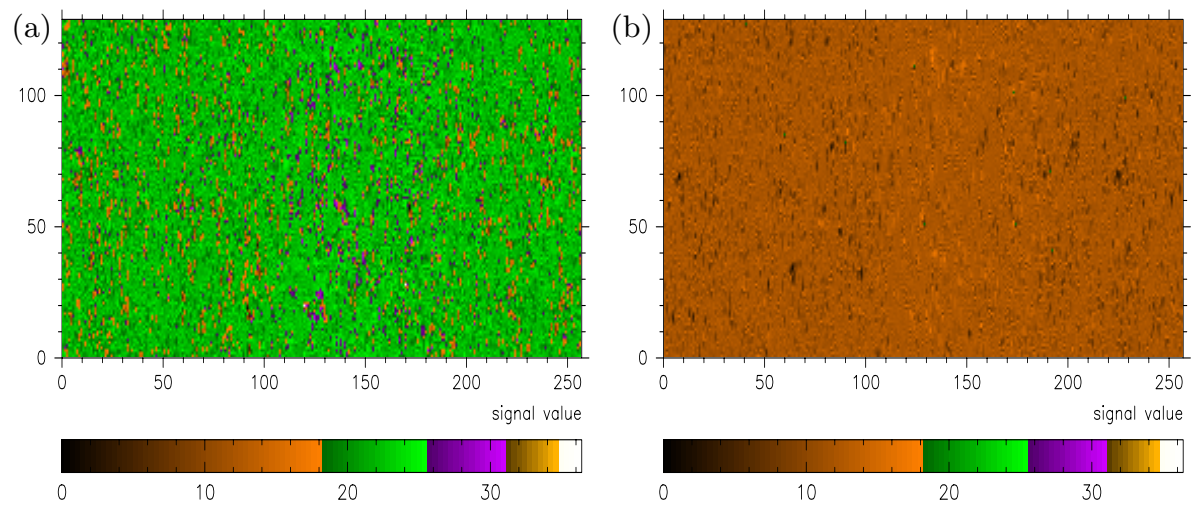

Fig. 3. Difference images generated by subtracting the reconstructed images using the unaligned (not shown) and the aligned pre-processed POCS method shown in Figure 2(d) from the true image Figure 2(a). Since differences between the reconstructed images were barely visible, the difference images are plotted on a discrete and logarithmic scale. Compared to the aligned counterpart (b) the difference image generated by the unaligned pre-processed POCS method (a) displays broad artifacts and also spurious high signal intensity changes.

Figure 3 compares difference images of the pre-processed POCS method using not-aligned (a) and aligned (b) pre-contrast amplitude filling by subtracting the reconstructed images from the true $T_{1}$-weighted post-contrast image shown in Figure 2(a). In Figure 4 signal intensity-time curves for the whole dynamic $T_{1}$ weighted imaging acquisition including two pre-contrast and five post-contrast volumes are displayed. The relative percentage signal increase during the first 90 secs after contrast injection compared to the pre-contrast volume was $245 \%$ which is a typical value for malignant tumors. In all cases the maximum signal enhancement is recovered within the calculated error. However, the characteristic washout behaviour (signal decrease immediately after early peak enhancement) can not be recovered by the Margosian or by the POCS method. In Figure 5, time series data for a $T_{2}^{*}$-weighted dynamic contrast enhanced single slice sequence 

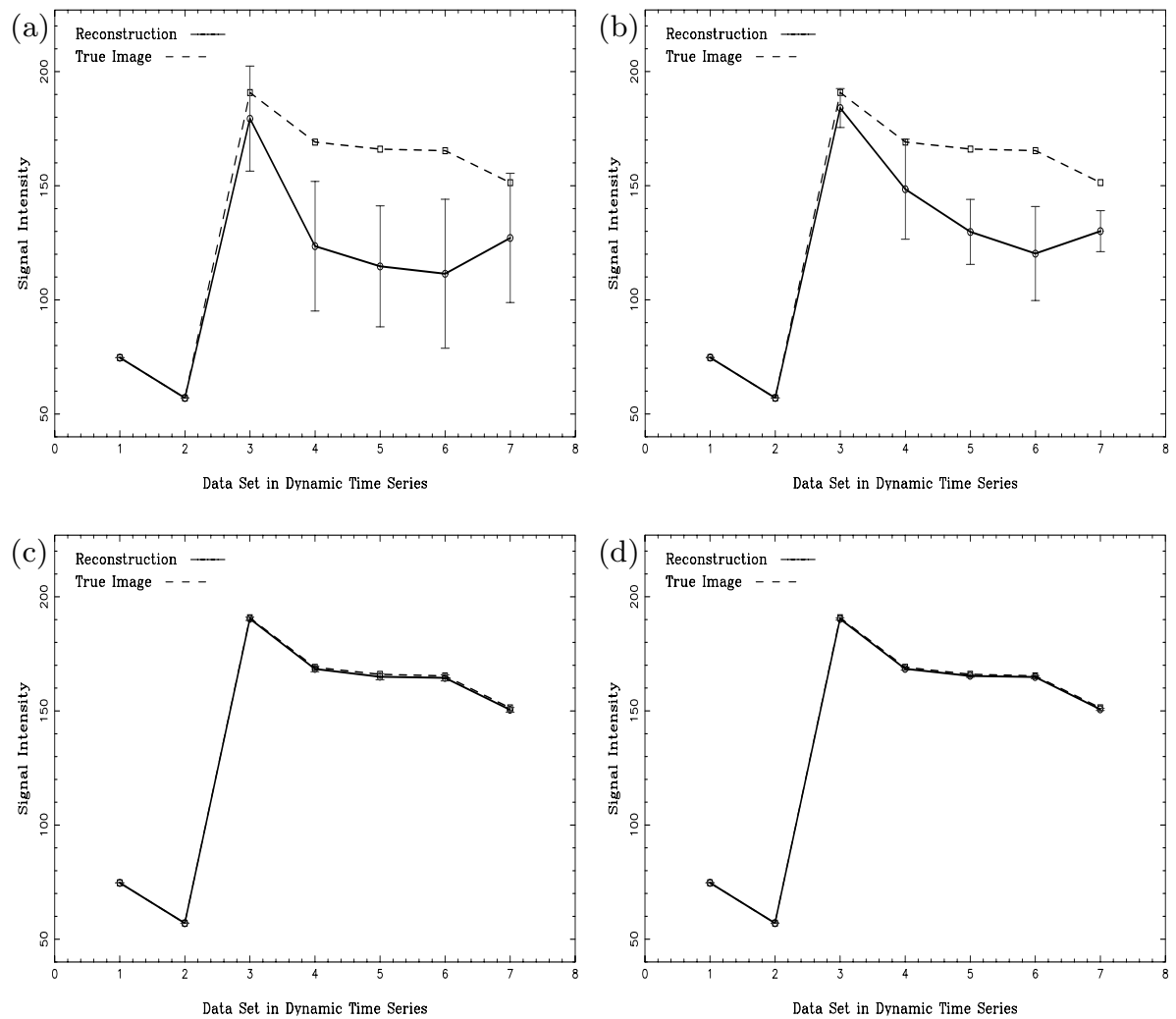

Fig. 4. $T_{1}$-weighted time series data for the $2 \mathrm{D}$ ROI shown in Figure 2(a). The Margosian method (a) and the POCS method (b) can not recover the correct temporal enhancement profile, although both reconstruction methods display the rapid enhancement for the first post-contrast volume. The results for the pre-processed POCS method are shown using unaligned (c) and aligned (d) precontrast amplitude data.

of the brain are displayed. The characteristic signal intensity loss for malignant tissue in the true signal-versus-time curve during the first 10 secs after contrast injection was $12 \%$ compared to the averaged baseline value. In Table 1 we summarize the results of all methods applied to brain and breast data calculating the time-averaged RMSE (11).

\section{Discussion and Conclusions}

We have applied a pre-processed half Fourier reconstruction method to different types of MRI time series data acquired dynamically following contrast agent administration. The method was evaluated on breast $T_{1}$-weighted image series' and brain $T_{2}^{*}$-weighted brain image series'. Unlike standard phase-constrained 

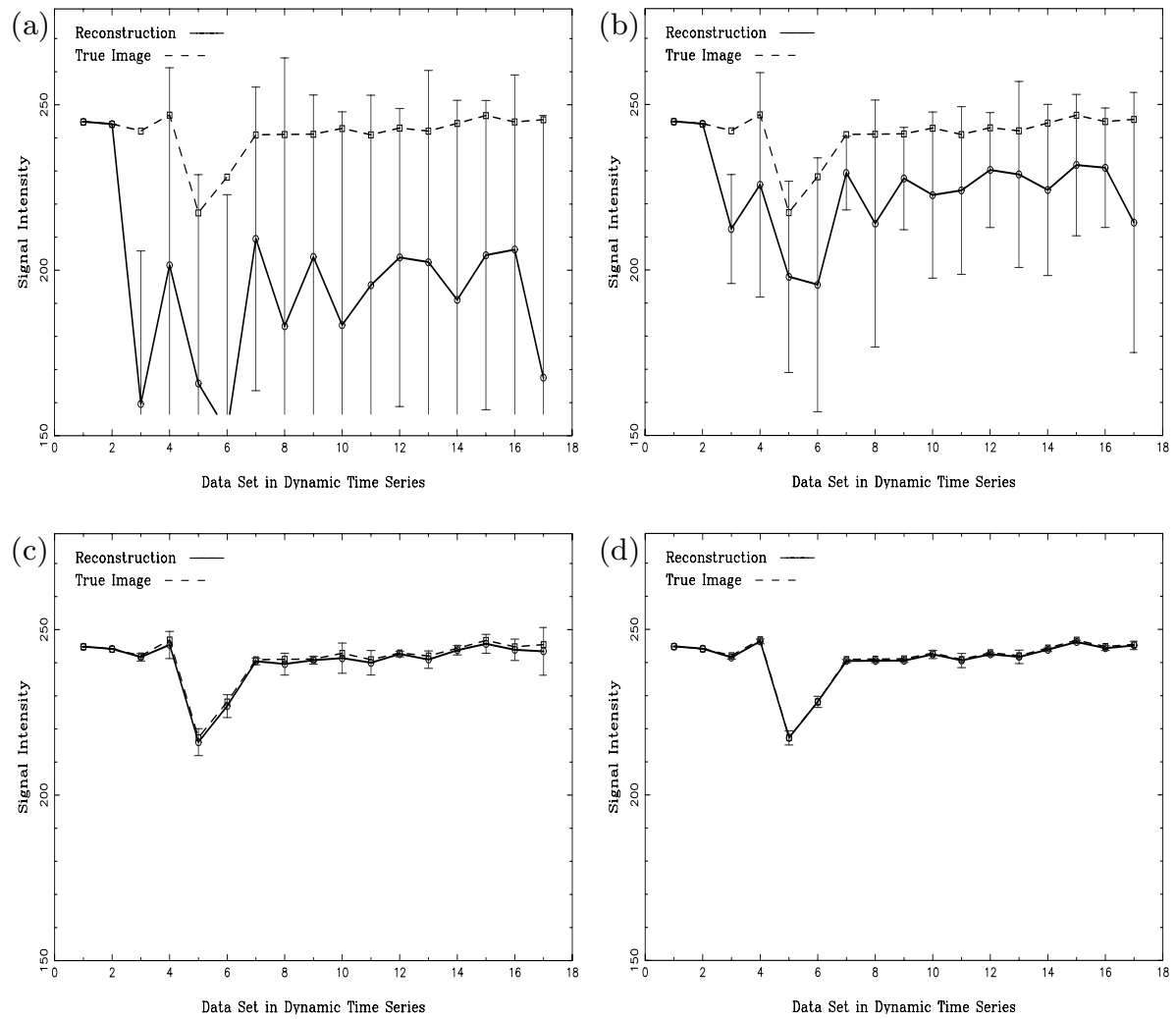

Fig. 5. Time series data for $T_{2}^{*}$-weighted first pass perfusion imaging of the brain. The perfusion drop after contrast agent injection has not been recovered using the Margosian method (a) and the POCS method (b). Both pre-processed POCS methods (c), (d) recover the perfusion drop.

reconstruction methods, the new algorithm, which is a variation of the POCS method, uses pre-contrast data to fill the unsampled part of Fourier space. Thus, Hamming filtering is not necessary to ensure a smooth transition in the centre of Fourier space. It was found that images reconstructed using the pre-processed POCS method have a far superior quality to those obtained using the standard methods. Furthermore, it was found that aligning the pre-contrast data with the post-contrast phase, yields images with an even better quality than those reconstructed without alignment. The image quality, as measured using the RMSE, improved by a factor of up to $90 \%$ on using the pre-contrast aligned data compared with that obtained using zero filling (see Table 1). In addition, it was found that a typical ROI in both breast and brain MR reconstructed images shows the same signal intensity-time curve profile as the original full Fourier images, with negligible error. This demonstrates that the algorithm has considerable potential in many MR imaging studies involving time series data. Future 
Table 1. The RMSE averaged over all data sets in the time series, for the whole image and a selected ROI. The percentages given in brackets below the absolute RMSE values measure the improvement on the RMSE using an aligned pre-contrast amplitude.

\begin{tabular}{lllll}
\hline $\begin{array}{l}\text { Type of } \\
\text { image data }\end{array}$ & $\begin{array}{l}\text { Magosian } \\
\text { method }\end{array}$ & $\begin{array}{l}\text { POCS } \\
\text { method }\end{array}$ & $\begin{array}{l}\text { POCS method using pre-contrast amplitude } \\
\text { not aligned }\end{array}$ & 1.29 \\
\hline $\begin{array}{l}256 \times 128 \times 64 \\
T_{1} \text { weighted } \\
\text { breast image }\end{array}$ & $\begin{array}{l}25.33 \\
(81.52 \%)\end{array}$ & $\begin{array}{l}15.49 \\
(69.79 \%)\end{array}$ & $\begin{array}{l}2.73 \\
(52.75 \%)\end{array}$ \\
Selected ROI & 27.77 & 14.85 & 1.12 & 0.56 \\
& $(97.98 \%)$ & $(96.23 \%)$ & $(50.0 \%)$ & \\
& & & & \\
$256 \times 128$ & 38.95 & 16.78 & 4.96 & 1.41 \\
$T_{2}^{*}$ weighted & $(95.96 \%)$ & $(91.6 \%)$ & $(71.57 \%)$ & 1.03 \\
brain image & & & & \\
Selected ROI & 58.71 & 25.47 & 2.98 & $(65.44 \%)$ \\
\hline
\end{tabular}

work will consider the impact of the location, size and type of the tumor on the numerical evaluation. Combined with half Fourier acquisition techniques and phase estimation algorithms, the method can result in an improvement in the temporal resolution by a factor of approximately two. This is certain to be beneficial in any fast imaging acquisition, particularly when the spatial resolution and/or coverage should not be compromised.

\section{Acknowledgments}

The authors are greatful to EPSRC GR/M52762 for their financial support of this work. The support of the Medical Research Council [MRC] and the Cancer Research Campaign [CRC] is grateful acknowledged.

\section{References}

1. Heywang, S.H., Hahn, D., Schmidt, H., Krischke, I., Eiermann, W., Bassermann, R., Lissner, J.: MR imaging of the breast using Gadolinium-DTPA. J. Comp. Ass. Tomography 10 (1986) 199-204

2. Kaiser, W.A., Zeitler, E.: MR imaging of the breast: fast imaging sequences with and without Gd-DTPA. Radiology 170 (1989) 681-686

3. Kvistad, K.A., Lundgren, S., Fjosne, H.E., Smenes, E., Smethurst, H.B., Haraldseth, O.:: Differentiating Benign And Malignant Breast Lesions with $T_{2}^{*}$-Weighted First Pass Perfusion Imaging. Acta Radiologica 40 (1999) 45-51

4. Lernevall, A.: Imaging of Axillary Lymph Nodes. Acta Oncologica 39 (2000) 277-281 
5. Liang, Z., Boada, F.E., Constable, R.T., Haacke, E.M., Lauterbur, P.C., Smith, M.R.: Constrained reconstruction methods in MR Imaging. Reviews of Magnetic Resonance in Medicine 4 (1992) 67-185

6. Press, W.H., Flannery, B.P., Teukolsky, S.A., Vetterling, W.T.: Numerical Recipes in C The Art of Scientific Computing. Cambridge University Press, Cambridge (1988)

7. Roberts, T.P.L., Chuang, N., Roberts, H.C.: Neuroimaging: do we really need new contrast agents for MRI? European Journal of Radiology 34 (2000) 166-178

8. Russ, J.C.: The Image Processing Handbook. CRC Press, Springer, Heidelberg (1998).

9. Stark, H.: Image Recovery: Theory and Application. Academic Press, New York (1987) 